\title{
Structural analysis of Bacillus megaterium KM spore peptidoglycan and its dynamics during germination
}

\author{
Abdelmadjid Atrih, ${ }^{1}$ Gerold Bacher, ${ }^{2}$ Roman Körner, ${ }^{3}$ Günter Allmaier ${ }^{2}$ \\ and Simon J. Foster ${ }^{1}$
}

Author for correspondence: Simon J. Foster. Tel: +44 114222 4411. Fax: +44 1142728697. e-mail: S.Foster $(\bar{a}$ sheffield.ac.uk

\footnotetext{
1 Department of Molecular Biology and Biotechnology, University of Sheffield, Firth Court Western Bank, Sheffield S10 2TN, UK

2 Institute for Analytical Chemistry, University of Vienna, Waehringer Str. 38, A-1090 Vienna, Austria

3 Department of Molecular Biology, University of Odense, DK-5230 Odense, Denmark
}

\begin{abstract}
The composition and structure of peptidoglycan from dormant spores of Bacillus megaterium KM and its dynamics during germination were investigated. Amino acid analysis and mass spectrometry identified 21 muropeptides resolved by reverse phase HPLC following digestion of peptidoglycan with Cellosyl. The basic structure of peptidoglycan in $B$. megaterium spores is similar to that of Bacillus subtilis: $\mathbf{4 4 \cdot 2} \%$ of muramic acid residues are substituted with $\delta$-lactam, $28.8 \%$ with single L-alanine, $25.1 \%$ with tetrapeptide and only $1.8 \%$ with tripeptide. The cross-linking index of the spore peptidoglycan, determined from muropeptides resolved by reverse phase HPLC, was $2.2 \%$ per muramic acid. Spore peptidoglycan contains $2.9 \%$ of muropeptides with unsubstituted $\boldsymbol{N}$-acety Imuramic acid. These muropeptides are likely to be intermediate products of $\delta$-lactam formation. Analysis of muropeptide dynamics during germination revealed the activity of at least two hydrolytic enzymes, an $\boldsymbol{N}$-acetylglucosaminidase and a lytic transglycosylase. A $4 \mathbf{M ~ L i C l ~ e x t r a c t ~ f r o m ~} \mathbf{3 0}$ min germinated spores of $B$. megaterium KM caused 'germination-like' changes to permeabilized spores of B. megaterium and B. subtilis but not those of a B. subtilis cwID mutant. Muropeptide analysis of the treated spores revealed the presence of products generated by the activity of a glucosaminidase.
\end{abstract}

Keywords: Bacillus megaterium KM, spores, peptidoglycan structure, germination

\section{INTRODUCTION}

The unique structure of spore cortex peptidoglycan is considered to play a major role in the maintenance of endospore heat resistance and dormancy (Ellar, 1978). The cortex retains core dehydration, which is initiated early during sporulation (Marquis et al., 1983). Earlier studies suggested that the overall structure of cortex peptidoglycan is conserved over many species including Bacillus sphaericus (Tipper \& Gauthier, 1972), Bacillus cereus, Bacillus megaterium, Bacillus stearothermophilus and Clostridium sporogenes (Warth \& Strominger, 1972). The spore cortex is characterized by the presence of spore-specific muramic $\delta$-lactam residues (Warth \& Strominger, 1969, 1972). The application of reverse phase (RP) HPLC combined with amino acid

Abbreviations: $A_{2} p m$, diaminopimelic acid; CID, collision-induced dissociation; ESI, electrospray ionization; FDNB, fluorodinitrobenzene; GSLE, germination-specific lytic enzyme; RP-HPLC, reverse phase HPLC. analysis and MS to study $B$. subtilis spore peptidoglycan has begun to reveal its fine structure and dynamics during differentiation (Atrih et al., 1996, 1998; Popham et al., 1996a). Almost $49 \%$ of muramic acid residues are substituted with $\delta$-lactam, $25 \cdot 7 \%$ by tetrapeptide and $23.3 \%$ by a single L-alanine residue. The cortex of Bacillus subtilis is loosely cross-linked, links occurring at only $2.9 \%$ of muramic acid residues (Atrih et al., 1996).

As the cortex maintains spore dormancy, its hydrolysis is essential for the germination process. Bacterial spore germination, defined as the irreversible loss of spore properties, is triggered by specific germinants. Although essential for outgrowth, cortex hydrolysis occurs as an intermediate germination event (Hsieh \& Vary, 1975; Popham et al., 1996b; Atrih et al., 1998). The $\delta$-lactam residues are not involved in maintaining dormancy but form a substrate recognition determinant for autolytic enzymes activated during germination, which are responsible for the hydrolysis of the cortex (Popham et al., 
1996b; Atrih et al., 1996, 1998). Although a number of cortex lytic enzymes have been isolated from germinated or physically broken spores of B. megaterium (Hsieh \& Vary 1975; Foster \& Johnstone 1987), very little is known about the exact nature and role of these enzymes in cortex hydrolysis. Hsieh \& Vary (1975) found both $\mathrm{N}$-acetylglucosaminidase and amidase activity in broken B. megaterium QMB 1551 spores. Foster \& Johnstone (1987) purified a germination-specific lytic enzyme (GSLE) from germinating spores of B. megaterium KM, which had been previously suggested to be an amidase (Johnstone \& Ellar, 1982). The GSLE was shown to hydrolyse only intact cortex containing $\delta$-lactam residues (Foster \& Johnstone, 1987).

This paper describes the analysis of B. megaterium $\mathrm{KM}$ spore peptidoglycan and its dynamics during germination. This analysis revealed conserved structural features with $B$. subtilis and the activity of germinationassociated enzymes.

\section{METHODS}

Spore preparation and germination. B. megaterium $\mathrm{KM}$ spores were prepared at $30^{\circ} \mathrm{C}$ in $\mathrm{CCY}$ medium as previously described (Foster \& Johnstone, 1987). Purified spores were heat-activated in distilled water at $70^{\circ} \mathrm{C}$ for $30 \mathrm{~min}$. Activated spores were immediately cooled on ice and used for germination experiments within $1 \mathrm{~h}$. The spores were suspended at a final concentration of $11 \mathrm{mg} \mathrm{m}^{-1}$ in potassium phosphate buffer ( $\mathrm{pH} \mathrm{7})$ and $\mathrm{KCl}$, each at a final concentration of $30 \mathrm{mM}$. Spores were then prewarmed for $15 \mathrm{~min}$ at $30^{\circ} \mathrm{C}$, before addition of L-alanine (final concn $1 \mathrm{mM}$ ). The decrease in $\mathrm{OD}_{600}$ and the loss of heat resistance during germination were monitored as previously described (Atrih et al., 1998).

Spore peptidoglycan structural analysis. Dormant and germinated spores were extracted and spore exudates prepared using the method previously described for B. subtilis (Atrih et al., 1998). Preparation, separation and analysis of muropeptides by RP-HPLC, amino acid analysis and MS were as described previously (Atrih et al., 1996, 1998). Nano electrospray ionization (ESI) mass spectra were acquired on a prototype Esquire ion trap mass spectrometer (BrukerFranzen Analytik) in the positive ion mode. The ion trap was operated at an estimated He gas pressure of $5 \times 10^{-3}$ mbar $(0.5$ $\mathrm{Pa}$ ). For low-energy collision-induced dissociation (CID) $\mathrm{MS}^{2}$ experiments, $\mathrm{He}$ was used as collision gas and resonance voltage pulses with amplitudes between 1 and $3 \mathrm{~V}$ (peak to peak) were applied across the endcap electrodes. The standard ESI source was replaced by a nano-electrospray ion source. Nano ESI fused silica needles were obtained from Protana. For all experiments, gold-plated needles were used. The lyophilized muropeptide fraction was dissolved in $10 \mu \mathrm{l}$ methanol/water $(50: 50, v / v)$. An aliquot of the sample solution $(0 \cdot 5-2 \mu \mathrm{l})$ was loaded into the needle with a gel loader pipette and a spray voltage of $700 \mathrm{~V}$ was applied.

Peptidoglycan cross-linking was determined by fluorodinitrobenzene (FDNB) as previously reported (Atrih et al., 1996) but without the TCA solubilization step (during spore extraction).

$\mathrm{LiCl}$ extraction of germinated spores. Spores of B. megaterium $\mathrm{KM}$ were permeabilized, heat-activated, germinated and $\mathrm{LiCl}$ extracted as previously described (Foster \& Johnstone, 1987). After dialysis of the extract the precipitate was recovered, redissolved and used as the source of lytic enzyme activity (Foster \& Johnstone, 1987).
Assay for lytic activity in the $\mathrm{LiCl}$ spore extract. Permeabilized spores $(5 \mathrm{mg})$ were incubated $\left(\right.$ at $30^{\circ} \mathrm{C}$ ) in the presence of $100 \mu \mathrm{l}$ spore extract in a final volume of $1 \mathrm{ml}$ in the buffer previously reported (Foster \& Johnstone, 1987). The reaction was stopped by boiling for $2 \mathrm{~min}$, the resulting material was digested with Cellosyl and muropeptides were analysed as above.

\section{RESULTS}

\section{Muropeptide analysis of dormant spores}

After spore extraction, Cellosyl digestion resulted in the solubilization of $97 \%$ of peptidoglycan as determined by the content of diaminopimelic acid $\left(\mathrm{A}_{2} \mathrm{pm}\right)$ in total permeabilized spores and Cellosyl-hydrolysed soluble and insoluble fractions. Soluble spore muropeptides obtained by Cellosyl digestion were separated by RPHPLC and a representative chromatogram is shown in Fig. 1(a). Purified muropeptides were subjected to amino acid and MS analyses and the results are shown in Table 1. The identity of muropeptides is shown in Table 2.

Muropeptide 10 had the same amino acid composition as muropeptide 11 (Fig. 1a, Tables 1 and 2; tetrasaccharide tetrapeptide), although it has a shorter retention time. MS analysis revealed an anomalous decrease in mass of $41 \cdot 6$, which suggests a possible de- $N$ acetylation of one amino sugar.

Muropeptides 13 and 14 (Fig. 1a) are respectively hexasaccharide tetrapeptide and hexasaccharide alanine, but each with an approximate mass difference of $+60 \mathrm{Da}$ (Tables 1 and 2). Analysis of muropeptide 13 by NMR indicated the presence of only one $\delta$-lactam and five $\mathrm{N}$-acetyl groups (result not shown). Based on NMR, MS and amino acid analyses, muropeptides 13 and 14 are respectively hexasaccharide tetrapeptide and hexasaccharide alanine with an unsubstituted $\mathrm{N}$-acetylmuramic acid (Tables 1 and 2).

Muropeptide 17 was previously found in B. subtilis 168 $\mathrm{HR}$ and suggested to be a hexasaccharide alanine with three acetyl groups and a lactam reduced to the minor form (Atrih et al., 1996). Further MS fragmentation analysis of muropeptide 17 , however, has revealed its primary structure (Fig. 2). Positive and negative ion matrix-assisted laser desorption ionization (MALDI) (see Table 1), as well as positive ion nano ESI $[\mathrm{M}+2 \mathrm{Na}-\mathrm{H}]^{+}$at $\mathrm{m} / z 1581 \cdot 2$ (observed value; $1581 \cdot 6$ calculated, monoisotopic) and $[\mathrm{M}+2 \mathrm{Na}]^{2+}$ at $m / z 791 \cdot 6$ (observed value; $791 \cdot 3$ calculated, monoisotopic) - mass spectra of muropeptide 17 exhibit abundant molecular ions. The low energy CID MS ${ }^{2}$ spectrum of the precursor ion $m / z 1582$ is shown in Fig. 2(a). The most abundant fragment ion $(m / z 1560)$ is related to the loss of one sodium ion from the $[\mathrm{M}+2 \mathrm{Na}-\mathrm{H}]^{+}$ion. From this fragment ion the loss of $\operatorname{MurNAc}(\mathrm{r})$-Ala gives an abundant ion at $m / z 1216$, followed by the loss of one GlcNAc moiety $(m / z 1013)$ and a subsequent loss of a MurNAc- $\delta$-lactam moiety $(m / z 798)$. This sequence of fragment ions in combination with the detected building blocks (after total hydrolysis of the fraction) characterizes the reduced end (generated by sodium borohydride 

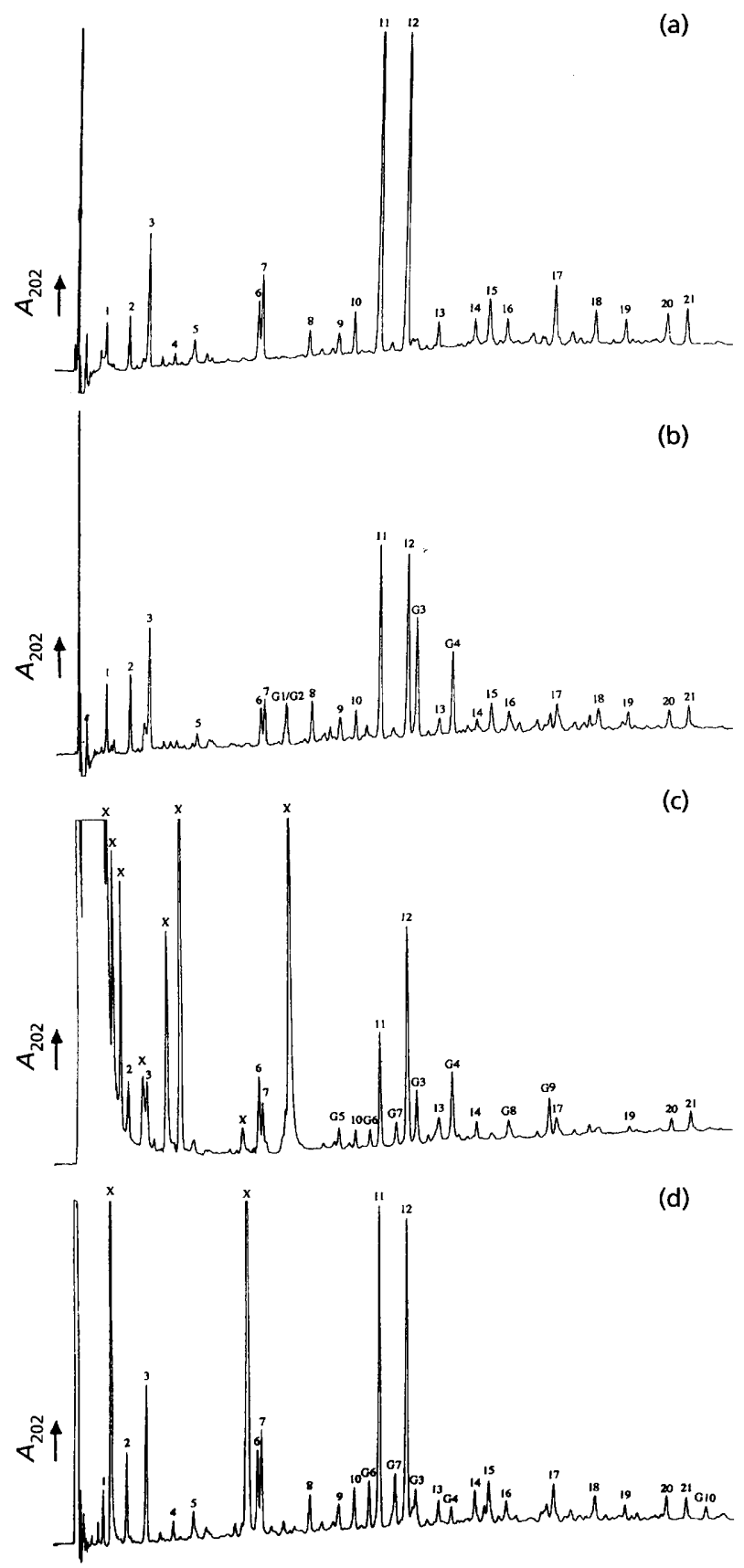

Fig. 1. RP-HPLC analysis of $B$. megaterium KM muropeptides of dormant spores and during germination. Cellosyl-digested muropeptides were separated by RP-HPLC and the $A_{202}$ of the eluates was monitored. (a) Dormant-spore-associated material; (b) germinated-spore-associated material; (c) germination exudate; (d) LiCl-extract-treated permeabilized spores. Muropeptides in (b) and (c) are from spores $45 \mathrm{~min}$ after addition of L-alanine. The peaks marked $X$ are primarily from non-peptidoglycan-derived material.

prior to HPLC separation) of the carbohydrate chain. The fragment ions at $m / z 1379$ (loss of GlcNAc directly from the precursor ion $\left.[\mathrm{M}+2 \mathrm{Na}-\mathrm{H}]^{+}\right), m / z 1033$ (loss of MurNAc-Ala) and $m / z 830$ (loss of GlcNAc) give the carbohydrate sequence from the non-reducing end of the hexasaccharide (Fig. 2b). By combining both sets of fragment ions, the mass spectrometric (MALDI and nano ESI) determined molecular mass and the building block composition (see Table 1), the structure has been elucidated unequivocally. Muropeptide 17 is hexasaccharide alanine with an extra alanine substitution (Fig. 2b). Likewise, muropeptide 16 is a hexasaccharide tetrapeptide with an extra alanine substitution (Tables 1 and 2).

From amino acid ratios, muropeptide 20 is an octasaccharide alanine. However, this muropeptide has an extra $88 \mathrm{Da}$ (Table 1). The difference in mass is that expected for an additional alanine substitution (as for muropeptides 16 and 17 ; Fig. 2 ) and de- $\mathrm{N}$-acetylation of an amino sugar (Tables 1 and 2).

The remaining muropeptides have been previously detected in B. subtilis spore cortex (Atrih et al., 1996), with muropeptide 11 (tetrasaccharide tetrapeptide) and 12 (tetrasaccharide alanine) (Fig. 1a; Table 2) accounting for $48 \%$ of the total muropeptides (Table 2). Muropeptides 1 and 8 have tripeptide side-chains and are likely to represent the primordial cell wall, together they make up $3.5 \%$ of the total muropeptides. Analysis of the overall composition of peptidoglycan from $B$. megaterium $\mathrm{KM}$ spores revealed that $44.2 \%$ of muramic acid residues are substituted with $\delta$-lactam, $28 \cdot 8 \%$ with single L-alanine, $25.1 \%$ with tetrapeptides and only $1 \cdot 8 \%$ with tripeptides.

The degree of cross-linking between glycan strands in spore cortex has previously been shown to be very low in B. subtilis (Atrih et al., 1996). The cross-linking index (percentage of possible residues forming cross-links) is only $8.7 \%$ per $\mathrm{A}_{2} \mathrm{pm}$ residue (calculated from muropeptides analysed by HPLC, Table 2 ). When calculated per muramic acid residue, the RP-HPLC data give a cross-linking index of $2 \cdot 2 \%$. The cross-linking index was determined independently by estimation of the amounts of $\mathrm{A}_{2} \mathrm{pm}$ present in acid hydrolysates, with and without the reaction of the free amino groups with FDNB (Atrih et al., 1996). An index of $24 \cdot 1 \%$ per $\mathrm{A}_{2} \mathrm{pm}$ residue was found. However, hydrolysis of the peptidoglycan by Cellosyl after FDNB treatment followed by amino acid analysis revealed that in the intact material almost $30 \%$ of the free amino groups in $\mathrm{A}_{2} \mathrm{pm}$ do not react with FDNB. This shows that the spore cortex is not freely accessible to FDNB and this will affect the results gained by this method. Therefore the crosslinking index calculated by the FDNB method should only be $16 \%$.

\section{Digestion of peptidoglycan by lysozyme}

B. megaterium cortex was digested with egg white lysozyme and the soluble material analysed by RPHPLC. Muropeptides 13, 14, 16 and 17 completely disappeared from the RP-HPLC profile of the lysozyme digest. Muropeptide 10 was also not present as the de$\mathrm{N}$-acetylation renders the adjacent bond resistant to lysozyme (Warth, 1978). Muropeptide 20, which is suggested to be octasaccharide alanine with an ad- 
Table 1. Calculated and observed $\mathrm{m} / \mathrm{z}$ values for sodiated and deprotonated molecular ions of $B$. megaterium $\mathrm{KM}$ spore peptidoglycan and muropeptide composition

\begin{tabular}{|c|c|c|c|c|c|c|c|c|c|c|c|}
\hline \multirow[t]{2}{*}{ Muropeptide* } & \multirow[t]{2}{*}{ Ion } & \multicolumn{2}{|c|}{$m / z$} & \multirow{2}{*}{$\begin{array}{c}\Delta m \\
(\mathrm{Da}) \dagger\end{array}$} & \multirow{2}{*}{$\begin{array}{l}\text { Error } \\
(\%) \neq\end{array}$} & \multicolumn{6}{|c|}{ Muropeptide composition (ratio) $\mathbb{S}$} \\
\hline & & Observed & Calculated & & & Glc & Mur & $\delta$-Mur & Glu & Ala & $\mathrm{A}_{2} \mathrm{pm}$ \\
\hline 1 & $\begin{array}{l}{[\mathrm{M}+\mathrm{Na}]^{+}} \\
{[\mathrm{M}-\mathrm{H}]^{-}}\end{array}$ & $\begin{array}{l}894 \cdot 6 \\
870 \cdot 4\end{array}$ & $\begin{array}{l}893 \cdot 9 . \\
869 \cdot 9\end{array}$ & $\begin{array}{l}0.7 \\
0.5\end{array}$ & $\begin{array}{l}0 \cdot 08 \\
0.06\end{array}$ & 1 & 1 & 0 & 1 & 1 & 1 \\
\hline 2 & $\begin{array}{l}{[\mathrm{M}+\mathrm{Na}]^{+}} \\
{[\mathrm{M}-\mathrm{H}]^{-}}\end{array}$ & $\begin{array}{l}592 \cdot 8 \\
568 \cdot 6\end{array}$ & $\begin{array}{l}592 \cdot 6 \\
568 \cdot 6\end{array}$ & $\begin{array}{l}0 \cdot 2 \\
0 \cdot 0\end{array}$ & $\begin{array}{l}0 \cdot 03 \\
0 \cdot 00\end{array}$ & 1 & 1 & 0 & 0 & 1 & 0 \\
\hline 3 & $\begin{array}{l}{[\mathrm{M}+\mathrm{H}]^{+}} \\
{[\mathrm{M}-\mathrm{H}]^{-}}\end{array}$ & $\begin{array}{l}943.5 \\
941 \cdot 9\end{array}$ & $\begin{array}{l}942 \cdot 9 \\
940 \cdot 9\end{array}$ & $\begin{array}{l}0 \cdot 6 \\
0 \cdot 2\end{array}$ & $\begin{array}{l}0 \cdot 06 \\
0 \cdot 02\end{array}$ & 1 & 1 & 0 & 1 & 2 & 1 \\
\hline 4 & $\begin{array}{l}{[\mathrm{M}+\mathrm{Na}]^{+}} \\
{[\mathrm{M}-\mathrm{H}]^{-}}\end{array}$ & $\begin{array}{l}1029 \cdot 8 \\
1005 \cdot 6\end{array}$ & $\begin{array}{r}1011 \cdot 0 \\
987 \cdot 0\end{array}$ & $\begin{array}{l}18 \cdot 8 \\
18 \cdot 6\end{array}$ & & 2 & 1 & 1 & 0 & 1 & 0 \\
\hline 5 & $\begin{array}{l}{[\mathrm{M}+\mathrm{Na}]^{+}} \\
{[\mathrm{M}-\mathrm{H}]^{-}}\end{array}$ & $\begin{array}{l}1402 \cdot 5 \\
1378 \cdot 3\end{array}$ & $\begin{array}{l}1383 \cdot 3 \\
1359 \cdot 3\end{array}$ & $\begin{array}{l}19 \cdot 2 \\
19 \cdot 0\end{array}$ & & 2 & 1 & 1 & 1 & 2 & 1 \\
\hline 6 & $\begin{array}{l}{[\mathrm{M}+\mathrm{Na}]^{+}} \\
{[\mathrm{M}-\mathrm{H}]^{-}}\end{array}$ & $\begin{array}{l}997 \cdot 4 \\
973.5\end{array}$ & $\begin{array}{r}1011 \cdot 0 \\
987 \cdot 0\end{array}$ & $\begin{array}{l}-13 \cdot 3 \\
-13 \cdot 5\end{array}$ & & 2 & 1 & 1 & 0 & 1 & 0 \\
\hline 7 & $\begin{array}{l}{[\mathrm{M}+\mathrm{Na}]^{+}} \\
{[\mathrm{M}-\mathrm{H}]^{-}}\end{array}$ & $\begin{array}{l}1369 \cdot 7 \\
1346 \cdot 0\end{array}$ & $\begin{array}{l}1383 \cdot 3 \\
1359 \cdot 3\end{array}$ & $\begin{array}{l}-13 \cdot 6 \\
-13 \cdot 3\end{array}$ & & 2 & 1 & 1 & 1 & 2 & 1 \\
\hline 8 & $\begin{array}{l}{[\mathrm{M}+\mathrm{Na}]^{+}} \\
{[\mathrm{M}-\mathrm{H}]^{-}}\end{array}$ & $\begin{array}{l}1818 \cdot 3 \\
1793 \cdot 5\end{array}$ & $\begin{array}{l}1817 \cdot 8 \\
1793 \cdot 8\end{array}$ & $\begin{array}{r}0.5 \\
-0.3\end{array}$ & $\begin{array}{r}0.02 \\
-0.01\end{array}$ & 2 & 2 & 0 & 2 & 3 & 2 \\
\hline 9 & $\begin{array}{l}{[\mathrm{M}+\mathrm{Na}]^{+}} \\
{[\mathrm{M}-\mathrm{H}]^{-}}\end{array}$ & $\begin{array}{l}1888 \cdot 7 \\
1864 \cdot 6\end{array}$ & $\begin{array}{l}1888 \cdot 9 \\
1864 \cdot 9\end{array}$ & $\begin{array}{l}-0 \cdot 2 \\
-0 \cdot 3\end{array}$ & $\begin{array}{l}-0.01 \\
-0.01\end{array}$ & 2 & 2 & 0 & 2 & 4 & 2 \\
\hline 10 & $\begin{array}{l}{[\mathrm{M}+\mathrm{H}]^{+}} \\
{[\mathrm{M}-\mathrm{H}]^{-}}\end{array}$ & $\begin{array}{l}1319 \cdot 9 \\
1317 \cdot 5\end{array}$ & $\begin{array}{l}1361 \cdot 3 \\
1359 \cdot 3\end{array}$ & $\begin{array}{l}-41 \cdot 4 \\
-41 \cdot 8\end{array}$ & & 2 & 1 & 1 & 1 & 2 & 1 \\
\hline 11 & $\begin{array}{l}{[\mathrm{M}+\mathrm{Na}]^{+}} \\
{[\mathrm{M}-\mathrm{H}]^{-}}\end{array}$ & $\begin{array}{l}1384 \cdot 0 \\
1361 \cdot 2\end{array}$ & $\begin{array}{l}1383 \cdot 3 \\
1359 \cdot 3\end{array}$ & $\begin{array}{l}0 \cdot 7 \\
1 \cdot 9\end{array}$ & $\begin{array}{l}0 \cdot 05 \\
0 \cdot 13\end{array}$ & 2 & 1 & 1 & 1 & 2 & 1 \\
\hline 12 & $\begin{array}{l}{[\mathrm{M}+\mathrm{Na}]^{+}} \\
{[\mathrm{M}-\mathrm{H}]^{-}}\end{array}$ & $\begin{array}{r}1011 \cdot 8 \\
987 \cdot 6\end{array}$ & $\begin{array}{r}1011 \cdot 0 \\
987 \cdot 0\end{array}$ & $\begin{array}{l}0 \cdot 8 \\
0 \cdot 6\end{array}$ & $\begin{array}{l}0 \cdot 07 \\
0 \cdot 06\end{array}$ & 2 & 1 & 1 & 0 & 1 & 0 \\
\hline 13 & $\begin{array}{l}{[\mathrm{M}+\mathrm{Na}]^{+}} \\
{[\mathrm{M}-\mathrm{H}]^{-}}\end{array}$ & $\begin{array}{l}1860 \cdot 6 \\
1838 \cdot 1\end{array}$ & $\begin{array}{l}1801 \cdot 7 \\
1777 \cdot 7\end{array}$ & $\begin{array}{l}58 \cdot 9 \\
60 \cdot 4\end{array}$ & & 3 & 2 & 1 & 1 & 2 & 1 \\
\hline 14 & $\begin{array}{l}{[\mathrm{M}+\mathrm{Na}]^{+}} \\
{[\mathrm{M}-\mathrm{H}]^{-}}\end{array}$ & $\begin{array}{l}1491 \cdot 0 \\
1466 \cdot 0\end{array}$ & $\begin{array}{l}1429 \cdot 4 \\
1405 \cdot 4\end{array}$ & $\begin{array}{l}61 \cdot 6 \\
60 \cdot 6\end{array}$ & & 3 & 2 & 1 & 0 & 1 & 0 \\
\hline 15 & $\begin{array}{l}{[\mathrm{M}+\mathrm{Na}]^{+}} \\
{[\mathrm{M}-\mathrm{H}]^{-}}\end{array}$ & $\begin{array}{l}2308 \cdot 3 \\
2282 \cdot 9\end{array}$ & $\begin{array}{l}2307 \cdot 2 \\
2283 \cdot 2\end{array}$ & $\begin{array}{r}1 \cdot 1 \\
-0 \cdot 3\end{array}$ & $\begin{array}{r}0 \cdot 04 \\
-0 \cdot 01\end{array}$ & 3 & 2 & 1 & 2 & 4 & 2 \\
\hline 16 & $\begin{array}{l}{[\mathrm{M}+\mathrm{Na}]^{+}} \\
{[\mathrm{M}-\mathrm{H}]^{-}}\end{array}$ & $\begin{array}{l}1930 \cdot 8 \\
1907 \cdot 8\end{array}$ & $\begin{array}{l}1801 \cdot 7 \\
1777 \cdot 7\end{array}$ & $\begin{array}{l}129 \cdot 1 \\
130 \cdot 1\end{array}$ & & 3 & 2 & 1 & 1 & 3 & 1 \\
\hline 17 & $\begin{array}{l}{[\mathrm{M}+\mathrm{Na}]^{+}} \\
{[\mathrm{M}-\mathrm{H}]^{-}}\end{array}$ & $\begin{array}{l}1560 \cdot 3 \\
1535 \cdot 7\end{array}$ & $\begin{array}{l}1429 \cdot 4 \\
1405 \cdot 4\end{array}$ & $\begin{array}{l}130 \cdot 9 \\
130 \cdot 3\end{array}$ & & 3 & 2 & 1 & 0 & 2 & 0 \\
\hline 18 & $\begin{array}{l}{[\mathrm{M}+\mathrm{Na}]^{+}} \\
{[\mathrm{M}-\mathrm{H}]^{-}}\end{array}$ & $\begin{array}{l}2724 \cdot 4 \\
2699 \cdot 9\end{array}$ & $\begin{array}{l}2725 \cdot 6 \\
2701 \cdot 6\end{array}$ & $\begin{array}{l}-1 \cdot 2 \\
-1 \cdot 7\end{array}$ & $\begin{array}{l}-0.04 \\
-0.06\end{array}$ & 4 & 2 & 2 & 2 & 4 & 2 \\
\hline 19 & $\begin{array}{l}{[\mathrm{M}+\mathrm{Na}]^{+}} \\
{[\mathrm{M}-\mathrm{H}]^{-}}\end{array}$ & $\begin{array}{l}1801 \cdot 0 \\
1777 \cdot 3\end{array}$ & $\begin{array}{l}1801 \cdot 7 \\
1777 \cdot 7\end{array}$ & $\begin{array}{l}-0 \cdot 7 \\
-0.4\end{array}$ & $\begin{array}{l}-0.03 \\
-0.02\end{array}$ & 3 & 1 & 2 & 1 & 2 & 1 \\
\hline 20 & $\begin{array}{l}{[\mathrm{M}+\mathrm{Na}]^{+}} \\
{[\mathrm{M}-\mathrm{H}]^{-}}\end{array}$ & $\begin{array}{l}1936 \cdot 5 \\
1912.5\end{array}$ & $\begin{array}{l}1847 \cdot 8 \\
1823 \cdot 8\end{array}$ & $\begin{array}{l}88 \cdot 7 \\
88 \cdot 7\end{array}$ & & 4 & 2 & 2 & 0 & 2 & 0 \\
\hline 21 & $\begin{array}{l}{[\mathrm{M}+\mathrm{Na}]^{+}} \\
{[\mathrm{M}-\mathrm{H}]^{-}} \\
\end{array}$ & $\begin{array}{l}1430 \cdot 9 \\
1406 \cdot 1 \\
\end{array}$ & $\begin{array}{l}1429 \cdot 4 \\
1405 \cdot 4 \\
\end{array}$ & $\begin{array}{l}1 \cdot 5 \\
0 \cdot 7 \\
\end{array}$ & $\begin{array}{l}0 \cdot 10 \\
0 \cdot 04 \\
\end{array}$ & 3 & 1 & 2 & 0 & 1 & 0 \\
\hline
\end{tabular}


Table 1 (cont.)

\begin{tabular}{|c|c|c|c|c|c|c|c|c|c|c|c|}
\hline \multirow[t]{2}{*}{ Muropeptide* } & \multirow[t]{2}{*}{ Ion } & \multicolumn{2}{|c|}{$m / z$} & \multirow{2}{*}{$\begin{array}{c}\Delta \boldsymbol{m} \\
(\mathrm{Da}) \dagger\end{array}$} & \multirow{2}{*}{$\begin{array}{l}\text { Error } \\
(\%) \ddagger\end{array}$} & \multicolumn{6}{|c|}{ Muropeptide composition (ratio) $\mathbb{S}$} \\
\hline & & Observed & Calculated & & & Glc & Mur & $\delta$-Mur & Glu & Ala & $\mathrm{A}_{2} \mathrm{pm}$ \\
\hline G1 & $\begin{array}{l}{[\mathrm{M}+\mathrm{Na}]^{+}} \\
{[\mathrm{M}-\mathrm{H}]^{-}}\end{array}$ & $\begin{array}{l}996 \cdot 4 \\
972 \cdot 5\end{array}$ & $\begin{array}{r}1011 \cdot 0 \\
987 \cdot 0\end{array}$ & $\begin{array}{l}-14 \cdot 6 \\
-14 \cdot 5\end{array}$ & & 2 & 1 & 1 & 0 & 1 & 0 \\
\hline G2 & $\begin{array}{l}{[\mathrm{M}+\mathrm{Na}]^{+}} \\
{[\mathrm{M}-\mathrm{H}]^{-}}\end{array}$ & $\begin{array}{l}1369 \cdot 6 \\
1345 \cdot 7\end{array}$ & $\begin{array}{l}1383 \cdot 3 \\
1359 \cdot 3\end{array}$ & $\begin{array}{l}-13 \cdot 7 \\
-13 \cdot 6\end{array}$ & & 2 & 1 & 1 & 1 & 2 & 1 \\
\hline G3 & $\begin{array}{l}{[\mathrm{M}+\mathrm{Na}]^{+}} \\
{[\mathrm{M}-\mathrm{H}]^{-}}\end{array}$ & $\begin{array}{l}1384 \cdot 4 \\
1359 \cdot 6\end{array}$ & $\begin{array}{l}1383 \cdot 3 \\
1359 \cdot 3\end{array}$ & $\begin{array}{l}1 \cdot 1 \\
0 \cdot 3\end{array}$ & $\begin{array}{l}0.07 \\
0.02\end{array}$ & 2 & 1 & 1 & 1 & 2 & 1 \\
\hline G4 & $\begin{array}{l}{[\mathrm{M}+\mathrm{Na}]^{+}} \\
{[\mathrm{M}-\mathrm{H}]^{-}}\end{array}$ & $\begin{array}{r}1009 \cdot 6 \\
986 \cdot 3\end{array}$ & $\begin{array}{r}1011 \cdot 0 \\
987 \cdot 0\end{array}$ & $\begin{array}{l}-1.4 \\
-0.7\end{array}$ & $\begin{array}{l}-0 \cdot 13 \\
-0 \cdot 07\end{array}$ & 2 & 1 & 1 & 0 & 1 & 0 \\
\hline G5 & $\begin{array}{l}{[\mathrm{M}+\mathrm{H}]^{+}} \\
{[\mathrm{M}-\mathrm{H}]^{-}}\end{array}$ & $\begin{array}{l}947 \cdot 3 \\
945 \cdot 5\end{array}$ & $\begin{array}{l}989 \cdot 0 \\
987 \cdot 0\end{array}$ & $\begin{array}{l}-41 \cdot 7 \\
-41 \cdot 5\end{array}$ & & 2 & 1 & 1 & 0 & 1 & 0 \\
\hline G6 & $\begin{array}{l}{[\mathrm{M}+\mathrm{H}]^{+}} \\
{[\mathrm{M}-\mathrm{H}]^{-}}\end{array}$ & $\begin{array}{l}1158 \cdot 6 \\
1156 \cdot 1\end{array}$ & $\begin{array}{l}1361 \cdot 3 \\
1359 \cdot 3\end{array}$ & $\begin{array}{l}-202 \cdot 7 \\
-203 \cdot 2\end{array}$ & & 1 & 1 & 1 & 1 & 2 & 1 \\
\hline G7 & $\begin{array}{l}{[\mathrm{M}+\mathrm{H}]^{+}} \\
{[\mathrm{M}-\mathrm{H}]^{-}}\end{array}$ & $\begin{array}{l}785 \cdot 9 \\
783 \cdot 3\end{array}$ & $\begin{array}{l}989 \cdot 0 \\
987 \cdot 0\end{array}$ & $\begin{array}{l}-203 \cdot 1 \\
-203 \cdot 7\end{array}$ & & 1 & 1 & 1 & 0 & 1 & 0 \\
\hline G8 & $\begin{array}{l}{[\mathrm{M}+\mathrm{Na}]^{+}} \\
{[\mathrm{M}-\mathrm{H}]^{-}}\end{array}$ & $\begin{array}{l}1363 \cdot 4 \\
1339 \cdot 1\end{array}$ & $\begin{array}{l}1383 \cdot 3 \\
1359 \cdot 3\end{array}$ & $\begin{array}{l}-19.9 \\
-20 \cdot 2\end{array}$ & & 2 & 1 & 1 & 1 & 2 & 1 \\
\hline G9 & $\begin{array}{l}{[\mathrm{M}+\mathrm{Na}]^{+}} \\
{[\mathrm{M}-\mathrm{H}]^{-}}\end{array}$ & $\begin{array}{l}991 \cdot 5 \\
967 \cdot 3\end{array}$ & $\begin{array}{r}1011 \cdot 0 \\
987 \cdot 0\end{array}$ & $\begin{array}{l}-19 \cdot 5 \\
-19 \cdot 7\end{array}$ & & 2 & 1 & 1 & 0 & 1 & 0 \\
\hline G10 & $\begin{array}{l}{[\mathrm{M}+\mathrm{Na}]^{+}} \\
{[\mathrm{M}-\mathrm{H}]^{-}}\end{array}$ & $\begin{array}{l}1227 \cdot 7 \\
1203 \cdot 2\end{array}$ & $\begin{array}{l}1429 \cdot 4 \\
1405 \cdot 4\end{array}$ & $\begin{array}{l}-201 \cdot 7 \\
-202 \cdot 2\end{array}$ & & 2 & 1 & 2 & 0 & 1 & 0 \\
\hline
\end{tabular}

* Muropeptides are numbered as indicated in Fig. 1.

† Difference between observed and calculated sodiated or deprotonated molecular mass values. Boldface characters denote deviations where the calculated values are the most likely combinations of the substituent components.

$\neq$ Calculated as [(observed mass - calculated mass)/calculated mass] $\times 100$.

$\$$ Glc, $N$-acetylglucosamine; Mur, $N$-acetylmuramic acid; $\delta$-Mur, muramic $\delta$-lactam.

ditional alanine substitution and a de- $\mathrm{N}$-acetylation was present after lysozyme digestion. Thus it is possible that de- $N$-acetylation occurs on a glucosamine (Warth, 1978) adjacent to the first $\mathrm{N}$-acetylmuramic acid substituted with single L-alanine.

\section{Analysis of peptidoglycan structural dynamics during germination}

Spore-associated material. The RP-HPLC profile of muropeptides from 45 min germinated spores is shown in Fig. 1 (b). The major germination-associated changes in the muropeptide profile are the decrease in muropeptides containing $\delta$-lactam and the appearance of four novel muropeptides. These novel muropeptides, termed G1, G2, G3 and G4 (Fig. 1b), co-eluted with muropeptides G1-G4 from germinated spores of B. subtilis (results not shown) (Atrih et al., 1998). Amino acid analysis and MS confirmed their identity with the B. subtilis germinationassociated muropeptides (Tables 1 and 2). After 45 min germination, these muropeptides represent $20.6 \%$ of the total in the spore-associated material. The muropeptides contain the novel germination-associated modification noted in B. subtilis and proposed to be due to epimerase activity (Atrih et al., 1998). The ratio of disaccharide tetrapeptide to that of disaccharide alanine in dormant spores $(1 \cdot 7: 1)$ changes during germination to become $1 \cdot 1: 1$ after $45 \mathrm{~min}$. Indeed, disaccharide tetrapeptide (muropeptide 3) decreases much faster than disaccharide alanine (muropeptide 2) as can be seen in Fig. 1(a,b). The cross-linked muropeptides containing $\delta$-lactam decrease at the same rate and their amounts are still significant after 45 min germination (muropeptides 15 and 18; Fig. 1a, b; Table 2).

Germination exudate material. The RP-HPLC profile of the germination exudate digested with Cellosyl and reduced with sodium borohydride is shown in Fig. 1(c). Muropeptides G1-G4 were detected in both the sporeassociated and germination exudate material. On the presented trace, muropeptides G1 and G2 (Fig. 1c) are hidden below non-peptidoglycan material. Muropeptides G5-G9 are germination-exudate-specific. Amino acid analysis and MS of muropeptide G5 indicated that this is tetrasaccharide alanine with a mass difference of $-41.6 \mathrm{Da}$, which corresponds to an acetyl group. Based on amino acid analysis and MS results, muropeptides G6 and G7 (Fig. 1c) were identified 
Table 2. B. megaterium KM muropeptide identities and quantification

Peptidoglycan was from dormant spores (DM), or from spore-associated material (SAM) or germination exudate (GE) after 45 min germination

\begin{tabular}{|c|c|c|c|c|}
\hline \multirow[t]{2}{*}{ Muropeptide } & \multirow[t]{2}{*}{ Identity } & \multicolumn{3}{|c|}{ Molar percentage } \\
\hline & & $\mathrm{DM}$ & SAM & GE \\
\hline 1 & Disaccharide tripeptide & $2 \cdot 4$ & $5 \cdot 4$ & \\
\hline 2 & Disaccharide alanine & $6 \cdot 4$ & $10 \cdot 4$ & $9 \cdot 9$ \\
\hline 3 & Disaccharide tetrapeptide & $11 \cdot 1$ & $11 \cdot 6$ & $7 \cdot 5$ \\
\hline 4 & Tetrasaccharide alanine with an open lactam & $0 \cdot 6$ & & \\
\hline 5 & Tetrasaccharide tetrapeptide with an open lactam & $0 \cdot 6$ & $0 \cdot 5$ & \\
\hline 6 & Tetrasaccharide alanine with a reduced lactam & $7 \cdot 7$ & $5 \cdot 5$ & $13 \cdot 3$ \\
\hline 7 & $\begin{array}{l}\text { Tetrasaccharide tetrapeptide with a reduced } \\
\text { lactam }\end{array}$ & $5 \cdot 2$ & $3 \cdot 1$ & 4 \\
\hline 8 & Disaccharide tripeptide disaccharide tetrapeptide & $1 \cdot 2$ & $2 \cdot 3$ & \\
\hline 9 & $\begin{array}{l}\text { Disaccharide tetrapeptide disaccharide } \\
\text { tetrapeptide }\end{array}$ & $0 \cdot 5$ & $0 \cdot 8$ & \\
\hline 10 & $\begin{array}{l}\text { Tetrasaccharide tetrapeptide missing an } N \text {-acetyl } \\
\text { group }\end{array}$ & $1 \cdot 7$ & $1 \cdot 5$ & $1 \cdot 3$ \\
\hline 11 & Tetrasaccharide tetrapeptide & $18 \cdot 9$ & $10 \cdot 7$ & $8 \cdot 2$ \\
\hline 12 & Tetrasaccharide alanine & $29 \cdot 1$ & $18 \cdot 7$ & $26 \cdot 3$ \\
\hline 13 & $\begin{array}{l}\text { Hexasaccharide tetrapeptide with an } \\
\text { unsubstituted } N \text {-acetylmuramic acid }\end{array}$ & 1 & $0 \cdot 4$ & 0.7 \\
\hline 14 & $\begin{array}{l}\text { Hexasaccharide alanine with an unsubstituted } \\
\mathrm{N} \text {-acetylmuramic acid }\end{array}$ & $1 \cdot 9$ & $0 \cdot 4$ & $1 \cdot 7$ \\
\hline 15 & $\begin{array}{l}\text { Tetrasaccharide tetrapeptide disaccharide } \\
\text { tetrapeptide }\end{array}$ & $1 \cdot 9$ & $1 \cdot 4$ & \\
\hline 16 & $\begin{array}{l}\text { Hexasaccharide tetrapeptide with an additional } \\
\text { alanine }\end{array}$ & $1 \cdot 2$ & 0.9 & \\
\hline 17 & $\begin{array}{l}\text { Hexasaccharide alanine with an additional } \\
\text { alanine }\end{array}$ & $2 \cdot 5$ & $1 \cdot 3$ & $0 \cdot 4$ \\
\hline 18 & $\begin{array}{l}\text { Tetrasaccharide tetrapeptide tetrasaccharide } \\
\text { tetrapeptide }\end{array}$ & $0 \cdot 8$ & 0.5 & \\
\hline 19 & Hexasaccharide tetrapeptide & $1 \cdot 8$ & $1 \cdot 8$ & 0.5 \\
\hline 20 & $\begin{array}{l}\text { Octasaccharide alanine with an additional } \\
\text { alanine, missing an } N \text {-acetyl group }\end{array}$ & $1 \cdot 1$ & $1 \cdot 2$ & 0.6 \\
\hline 21 & Hexasaccharide alanine & $2 \cdot 3$ & $1 \cdot 0$ & $1 \cdot 6$ \\
\hline G1 & Tetrasaccharide alanine with a reduced lactam & & 4 & $\mathrm{ND}$ \\
\hline G2 & $\begin{array}{l}\text { Tetrasaccharide tetrapeptide with a reduced } \\
\text { lactam }\end{array}$ & & $1 \cdot 7$ & $\mathrm{ND}$ \\
\hline G3 & Tetrasaccharide tetrapeptide & & $6 \cdot 5$ & $3 \cdot 7$ \\
\hline G4 & Tetrasaccharide alanine & & $8 \cdot 4$ & $7 \cdot 5$ \\
\hline G5 & $\begin{array}{l}\text { Tetrasaccharide alanine missing an } N \text {-acetyl } \\
\text { group }\end{array}$ & & & $2 \cdot 4$ \\
\hline G6 & Trisaccharide tetrapeptide & & & $1 \cdot 2$ \\
\hline G7 & Trisaccharide alanine & & & $2 \cdot 4$ \\
\hline G8 & Anhydro-tetrasaccharide tetrapeptide & & & $1 \cdot 3$ \\
\hline G9 & Anhydro-tetrasaccharide alanine & & & $5 \cdot 4$ \\
\hline G10 & Pentasaccharide alanine & & & \\
\hline
\end{tabular}

ND, Not determined.

as trisaccharide tetrapeptide and trisaccharide alanine, respectively (Tables 1 and 2 ). These two products are present in small amounts $(3.6 \%)$ and are likely to occur as the result of glucosaminidase activity.
Muropeptides G8 and G9 were also detected in the germination exudate without sodium borohydride treatment (result not shown). They show similar properties to anhydro-muropeptides G9 and G10 found in the 


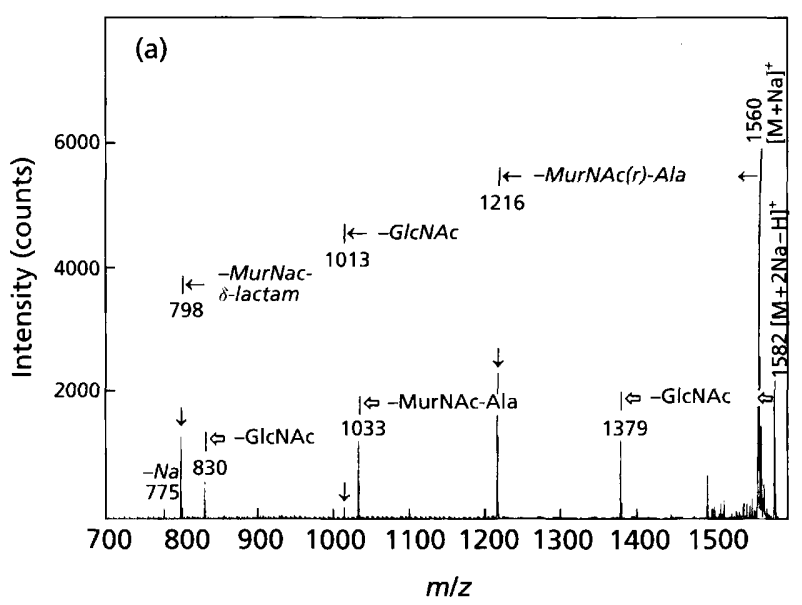

b)

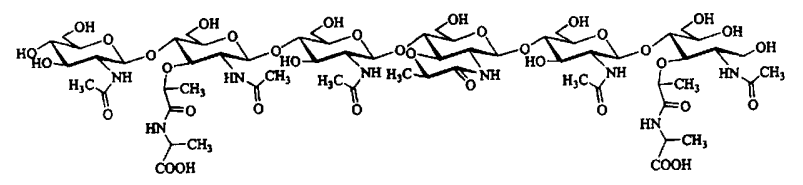

Fig. 2. Analysis of the structure of muropeptide 17. (a) Low energy $\mathrm{CID}$ product ion spectrum (MS ${ }^{2}$ ) of muropeptide 17 (precursor ion: $[\mathrm{M}+2 \mathrm{Na}-\mathrm{H}]^{+}, \mathrm{m} / \mathrm{z} 1582$, formed by nano ESI). GICNAC, $\mathrm{N}$-acetylglucosamine; MurNAC, $\mathrm{N}$-acetylmuramic acid; MurNAc- $\delta$-lactam, muramic- $\delta$-lactam; MurNAc-Ala, amino acid Ala linked to $\mathrm{N}$-acetylmuramic acid; MurNAc(r)-Ala, amino acid Ala linked to reduced $\mathrm{N}$-acetylmuramic acid. Labels in italics indicate the loss of various moieties starting from the reduced end of the carbohydrate chain. (b) Proposed chemical structure of muropeptide 17.

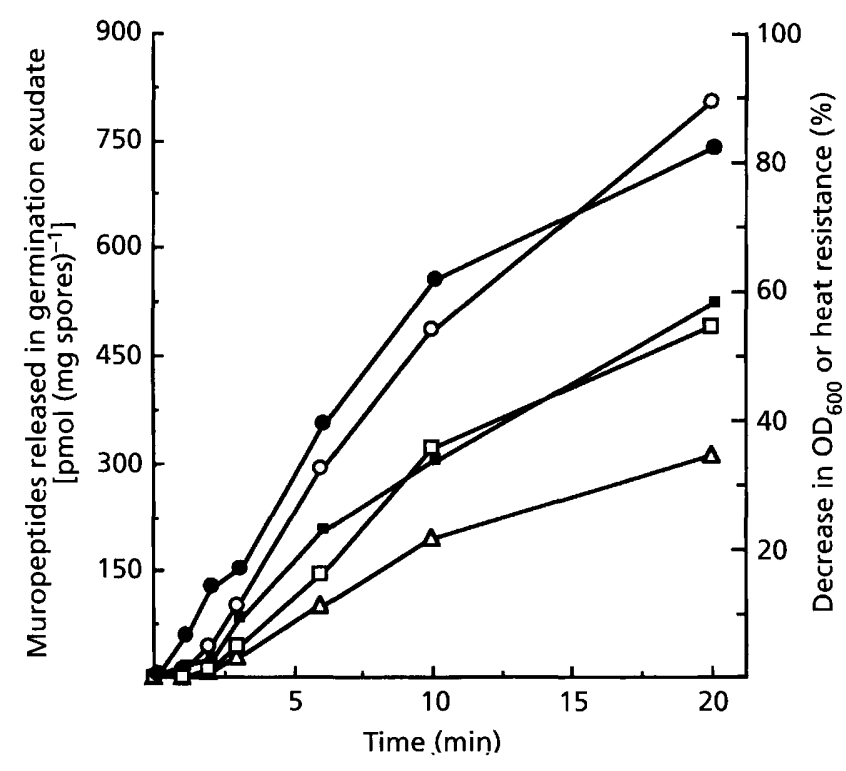

Fig. 3. Kinetics of biochemical events during germination of $B$. megaterium KM spores. Percentage loss of heat resistance; $\square$, percentage reduction in $\mathrm{OD}_{600} ; O$, amount of muropeptides G3 and G4; $\square$, amount of muropeptide G8 and G9; $\triangle$, amount of muropeptides G6 and G7. All muropeptides were measured from germination exudate samples. germination exudate of B. subtilis (Atrih et al., 1998). MS analysis confirmed their identities as anhydrotetrasaccharide tetrapeptide and anhydro-tetrasaccharide alanine, respectively (Tables 1 and 2). The two products represent $6.7 \%$ of the total muropeptides in the germination exudate (Table 2 ) and are produced due to the activity of a lytic transglycosylase. Peaks termed $\mathrm{X}$ are non-peptidoglycan products and some of them co-eluted with those already found in the $B$. subtilis germination exudate (Atrih et al., 1998).

A striking feature of the germination exudate muropeptides is the amount of tetrasaccharide alanine (muropeptide 12) compared to tetrasaccharide tetrapeptide (muropeptide 11). Indeed, the amount of muropeptide 12 is three times that of 11 , although their ratio is relatively constant in the germinated-spore-associated material compared to dormant spores (Table 2).

\section{Kinetics of peptidoglycan structural dynamics}

The first changes in spore-associated muropeptides were detected within $2 \mathrm{~min}$ of the initiation of germination (results not shown). However, the release of material in the exudate was detected as early as $1 \mathrm{~min}$, although only muropeptides G3 and G4 are released in quantifiable amounts at that time (Fig. 3). The decrease in optical density and the loss of heat resistance were detected within $1 \mathrm{~min}$ after addition of germinant Lalanine (Fig. 3).

Quantification of spore-associated material throughout germination revealed a faster release of muropeptides containing $\delta$-lactam compared to those without (result not shown). After $2 \mathrm{~h}$, muropeptides 1 and 8 became the dominant muropeptides in the spore-associated material, confirming their role as primordial cell wall (result not shown). Assay of the germination-associated novel muropeptides revealed the activities of enzymes occurring during germination. A relatively low activity of $\mathrm{N}$-acetylglucosaminidase (muropeptides $\mathrm{G} 6-\mathrm{G} 7$ ) was measured compared to putative epimerase (muropeptides G3-G4) and lytic transglycosylase (muropeptides G8-G9).

\section{Cortex lytic activity in a $\mathrm{LiCl}$ spore extract}

Previously a $\mathrm{LiCl}$ extract of germinating spores of $B$. megaterium $\mathrm{KM}$ has been used as a source of GSLE (Foster \& Johnstone, 1987, 1988). Permeabilized spores of B. megaterium KM, B. subtilis $\mathrm{HR}$ and B. subtilis AA107 ( $c w l D)$ were used as substrate to determine the lytic activities in an extract of germinating spores of $B$. megaterium KM. Both B. megaterium KM and B. subtilis HR permeabilized spores became phase-dark following overnight incubation with the extract, but not if it was previously boiled. The total (spore-associated and exudate) muropeptide profile of $B$. megaterium $\mathrm{KM}$ treated with the extract is shown in Fig. 1(d).

Surprisingly, the cross-linked muropeptides (peaks 15 and 18) do not decrease significantly in both $B$. 
megaterium KM (Fig. 1d) and B. subtilis (result not shown). Also, the ratio of muropeptides with tetrapeptide side-chains to those with single L-alanine is not affected. The muropeptides G6 and G7 are generated by an $\mathrm{N}$-acetylglucosaminidase. Likewise, the muropeptide G10 structure (determined by amino acid analysis and MS; Tables 1 and 2) confirms $\mathrm{N}$-acetylglucosaminidase as being the major autolytic enzyme present in the extract. Muropeptide G10 is a pentasaccharide alanine (Table 2). The putative extract-generated muropeptides (G3 and G4) are present in smaller amounts than the glucosaminidase products (G6, G7 and G10). The spores from the cwlD mutant (strain AA107) were unaffected by the extract and remained phase-bright with an unaltered muropeptide profile (result not shown).

\section{DISCUSSION}

The composition and structure of peptidoglycan from dormant spores of $B$. megaterium $\mathrm{KM}$ and its dynamics during germination were investigated. B. megaterium KM spore peptidoglycan has $76 \%$ of muropeptides in common with that of $B$. subtilis. The major difference is the presence of muropeptides with de- $N$-acetylation of one amino sugar, most likely the glucosamine as reported for the cortex of B. cereus (Warth, 1978), and muropeptides with unsubstituted $\mathrm{N}$-acetylmuramic acid. De- $\mathrm{N}$-acetylation of glucosamine is known to inhibit the action of lysozyme (Warth, 1978). Digestion of $B$. megaterium spore peptidoglycan with lysozyme confirms this property as muropeptide 10 disappears from the RP-HPLC trace. Muropeptides with unsubstituted $\mathrm{N}$-acetylmuramic acid would not be expected from the mechanism of peptidoglycan biosynthesis, which suggests the possible action of an amidase cleaving peptide side-chains during cortex maturation. These products could be intermediates in $\delta$ lactam formation. Indeed, it has long been suggested that formation of $\delta$-lactam is probably a two-step process (Tipper \& Gauthier, 1972). This would include the activity of an amidase cleaving peptide side-chains, followed by transacylase, which generates the lactam ring. In $B$. subtilis, the $c w l D$ gene encodes a putative sporulation-specific peptidoglycan hydrolase with sequence homology to other amidases (Sekiguchi et al., 1995). Inactivation of the gene resulted in spore peptidoglycan devoid of $\delta$-lactam (Sekiguchi et al., 1995; Atrih et al., 1996; Popham et al., 1996b). The presence of muropeptides with an extra alanine substitution suggests that the bond adjacent to the substitution is relatively resistant to Cellosyl. This may be due to a subtle modification, which alters muropeptide conformation.

The cross-linking index per muramic acid in spore peptidoglycan of $B$. megaterium $\mathrm{KM}$ is comparable to that of $B$. subtilis $\mathrm{HR}$, occurring respectively at $2 \cdot 2 \%$ and $2.9 \%$ (Atrih et al., 1996). The low cross-linking index therefore is a conserved feature in spore peptidoglycan structure. The low cross-linking will allow an apparent flexibility of spore peptidoglycan that may contribute to a defined architecture of this polymer, which is pivotal for maintenance of core dehydration and heat resistance. Recent studies have indeed shown that spores from a $\operatorname{dac} B$ mutant with a fivefold increase in peptidoglycan cross-linking are more heat-sensitive and spores are relatively unstable (Popham et al., 1995, 1996a; Atrih et al., 1996).

Analysis of peptidoglycan dynamics during germination showed a similar pattern of enzyme activities to those involved in cortex hydrolysis in B. subtilis (Atrih et al., 1998), and thus similar enzymes are likely to be involved. These include an $\mathrm{N}$-acetylglucosaminidase, a lytic transglycosylase and the activity generating the subtle modification suggested to be an epimerization of muramic acid (Atrih et al., 1998). From the analysis of muropeptides we have no evidence for an amidase activity in the form of amidase products. However, the analysis of the ratio of muropeptides with single L-alanine and tetrapeptide side-chains in the germination exudate clearly suggest that the amount of single L-alaninesubstituted muropeptides increased dramatically in the peptidoglycan fragments released in the exudate (Fig. 1c; Table 2). The shift in the ratio of these two types of muropeptides suggests the action of an amidase, which results in the decrease of tetrasaccharide side-chain and therefore a relative increase in L-alanine side-chain. An amidase activity has been detected in broken spores of $B$. megaterium (Hsieh \& Vary, 1975). Alternatively, an endopeptidase could cleave between alanine and glutamic acid and generate more muropeptides with Lalanine side-chain at the expense of muropeptides with tetrapeptide side-chain. An L-alanoyl-D-glutamate endopeptidase has been detected in sporulating cells of $B$. sphaericus (Kingan \& Ensign, 1968).

Earlier studies have suggested that the GSLE of $B$. megaterium $\mathrm{KM}$ is an amidase which cleaves crosslinked muropeptides and therefore removes the physical constraints on the core, allowing uptake of water (Johnstone \& Ellar, 1982; Foster \& Johnstone, 1987, 1990). The action of GSLE is associated with an increase of new cortical reducing groups in the form of $\delta$-lactam residues, which could be labelled with sodium borohydride (Foster \& Johnstone, 1987). In our current experiments, the major hydrolytic activity found in the germinated spore extract is an $\mathrm{N}$-acetylglucosaminidase. This suggests that the new $\delta$-lactam noted after the action of the GSLE and reduction with sodium borohydride is simply due to the opening of the peptidoglycan matrix, allowing the easy access of sodium borohydride to $\delta$-lactam moieties. The activity of the $N$-acetylglucosaminidase alone is sufficient to destroy the dormancy-maintaining function of the cortex. Warth (1978) suggested that $N$-acetylglucosaminidase is the main enzyme associated with cortex hydrolysis during germination by cleaving the long glycan strands.

Due to the presence of $\delta$-lactam, which is easily reduced by sodium borohydride (Warth \& Strominger, 1969; Atrih et al., 1996; Popham et al., 1996a), we believe that the classical methods for identification of lytic enzyme activities, using permeabilized spores as substrate (Moriyama et al., 1996; Foster \& Johnstone, 1987), 
should be viewed with caution. The low cross-linking index of spore peptidoglycan also suggests that the activity of amidases is very difficult to assay (Moriyama et al., 1996), especially if the activity is confined to the cross-linked muropeptides as it appears to be in $B$. subtilis (Atrih et al., 1998). Therefore, the use of RPHPLC is the method of choice for spore-associated activities.

A lytic transglycosylase activity has recently been described to be associated with germination of B. subtilis HR (Atrih et al., 1998). This may generate muropeptides that are recycled during outgrowth. This activity also occurs during germination of $B$. megaterium $\mathrm{KM}$, resulting in muropeptides G8 and G9. However, no lytic transglycosylase was detected in the germinated spore extract. This result suggests that the lytic transglycosylase is either inactive under our experimental conditions or it is released in the exudate along with the peptidoglycan fragments. The activity that is responsible for the subtle modification of muropeptides and suggested to be an epimerase (Atrih et al., 1998), is also found in the exudate. This activity will not in itself cause germination as it is not a lytic reaction.

The similarity in spore peptidoglycan structure and germination-associated structural dynamics between $B$. megaterium $\mathrm{KM}$ and $B$. subtilis suggests the involvement of a similar enzymic machinery in the biosynthesis and modification of the cortex and its hydrolysis during germination. The recent release of the B. subtilis genome sequence has allowed the identification of a number of genes homologous to known peptidoglycan hydrolases and biosynthetic enzymes of $B$. subtilis or other microorganisms (Kunst et al., 1997). The construction of mutants and analysis of peptidoglycan structure by RPHPLC technology is therefore a potential means to further our understanding of the unique role of the cortex in maintaining spore dormancy and the role of lytic enzymes in cortex hydrolysis during germination.

\section{ACKNOWLEDGEMENTS}

This work was supported by the BBSRC (A.A.), the Royal Society (S.J.F.), the Austrian Fonds zur Förderung der wissenschaftlichen Forschung (P11183 to G.A.) and the ARC Programme (Austria/UK travel fund). We thank P. Roepstorff for the generous access to the ion trap instrument, M. Williamson for NMR analysis and R. Marquardt for the gift of Cellosyl.

\section{REFERENCES}

Atrih, A., Zöllner, P., Allmaier, G. \& Foster, S. J. (1996). Structural analysis of Bacillus subtilis 168 endospore peptidoglycan and its role during differentiation. J Bacteriol 178, 6173-6183.

Atrih, A., Zöllner, P., Allmaier, G., Williamson, M. \& Foster, S. J. (1998). Peptidoglycan structural dynamics during germination of Bacillus subtilis 168 endospores. J Bacteriol 180, 4603-4612.

Ellar, D. J. (1978). Spore specific structures and their function. Symp Soc Gen Microbiol 28, 295-325.
Foster, S. J. \& Johnstone, K. (1987). Purification and properties of a germination-specific cortex lytic enzyme from spores of Bacillus megaterium KM. Biochem J 242, 573-579.

Foster, S. J. \& Johnstone, K. (1988). Germination-specific cortexlytic enzyme is activated during triggering of Bacillus megaterium KM spore germination. Mol Microbiol 2, 727-733.

Foster, S. J. \& Johnstone, K. (1990). Pulling the trigger: the mechanism of bacterial spore germination. Mol Microbiol 4, 1.37-141.

Hsieh, L. K. \& Vary, J. C. (1975). Germination and peptidoglycan solubilization in Bacillus megaterium spores. J Bacteriol 123, $463-470$.

Johnstone, K. \& Ellar, D. J. (1982). The role of cortex hydrolysis in the triggering of germination of Bacillus megaterium KM endospores. Biochim Biophys Acta 794, 185-191.

Kingan, S. L. \& Ensign, J. C. (1968). Isolation and characterization of three autolytic enzymes associated with sporulation of Bacillus thuringiensis. J Bacteriol 96, 629-638.

Kunst, F., Ogasawara, N. Moszer, I. \& 148 other authors (1997). The complete genome sequence of the gram-positive bacterium Bacillus subtilis. Nature 390, 249-256.

Marquis, R. E., Bender, G. R., Carstensen, E. L. \& Child, S. Z. (1983). Dielectric characterization of forespores isolated from Bacillus megaterium. J Bacteriol 153, 436-442.

Moriyama, R., Kudoh, S., Miyata, S., Nonobe, S., Hattori, A. \& Makino, S. (1996). A germination-specific spore cortex-lytic enzyme from Bacillus cereus spores: cloning and sequencing of the gene and molecular characterization of the enzyme. J Bacteriol 178, 5330-5332.

Popham, D. L., Illades-Aguiar, B. \& Setlow, P. (1995). The Bacillus subtilis $d a c B$ gene, encoding penicillin-binding protein $5^{*}$, is part of a three-gene operon required for proper spore cortex synthesis and spore core dehydration. J Bacteriol 177, 4721-4729.

Popham, D. L., Helin, J., Costello, C. E. \& Setlow, P. (1996a). Analysis of the peptidoglycan structure of Bacillus subtilis endospores. J Bacteriol 178, 6451-6458.

Popham, D. L., Helin, J., Costello, C. E. \& Setlow, P. (1996b). Muramic lactam in peptidoglycan of Bacillus subtilis spores is required for spore outgrowth but not spore dehydration or heat resistance. Proc Natl Acad Sci USA 93, 15405-15410.

Sekiguchi, J., Akeo, K., Yamamoto, H., Khasanov, F. K., Alonso, J. C. \& Kuroda, A. (1995). Nucleotide sequence and regulation of a new putative cell wall hydrolyse gene, cwlD, which affects germination in Bacillus subtilis. J Bacteriol 177, 5582-5589.

Tipper, D. J. \& Gauthier, J. J. (1972). In Spores V, Structure of the Bacterial Endospore, pp. 3-12. Edited by H. O. Halvorson, R. Hanson, \& L. L. Campbell. Washington, DC: American Society for Microbiology.

Warth, A. D. (1978). Molecular structure of the bacterial spore. Adv Microb Physiol 17, 1-47.

Warth, A. D. \& Strominger, J. L. (1969). Structure of bacterial spores: occurrence of the lactam of muramic acid. Proc Natl Acad Sci USA 64, 528-535.

Warth, A. D. \& Strominger, J. L. (1972). Structure of the peptidoglycan from spores of Bacillus subtilis. Biochemistry 11, 1389-1396.

Received 5 October 1998; revised 8 January 1999; accepted 20 January 1999. 